УДК 661.721.422

\title{
ВЛИЯНИЕ СОСТАВА ВОДОРОДСОДЕРЖАЩЕГО ГАЗА НА ВЫХОД МЕТАНОЛА
}

\author{
Загашвили Юрий Владимирович1, \\ y.zagashvili@yandex.ru
}

\author{
Кузьмин Алексей Михайлович2,3,4, \\ kuzmin.lex@gmail.com \\ 1000 «BTP», \\ Россия, 195297, г. Санкт-Петербург, Ольги Форш, 15 А, к. 1. \\ 2 Балтийский государственный технический университет «BOEHMEX» им. Д.Ф. Устинова, \\ Россия, 190005, г. Санкт-Петербург, 1-я Красноармейская, $1 / 21$ \\ 3 ООО «Генератор синтез-газа», \\ Россия, 195297, г. Санкт-Петербург, ул. Ольги Форш, 15, к. 1, кв. 49. \\ 4 Российская Академия Естественных Наук, \\ 190013, г. Санкт-Петербург, ул. Серпуховская, д. 38
}

\begin{abstract}
Актуальность исследования обусловлена отсутствием научно-обоснованных данных о выходе метанола из сырья в зависимости от типа используемого окислителя (кислород, обогащенный воздух, воздух) и оптимизации состава и параметров водородсодержащего газа по критериям отношения компонентов синтез-газа $\mathrm{H}_{2} / \mathrm{CO}$ и модуля (фракториала) водородсодержащего газа М для оптимального синтеза метанола. Проблема особенно важна для малотоннажных установок по производству метанола в промысловых условиях, работающих на забалластированном азотом водородсодержащем газе.

Цель: оценить влияние оптимизации состава водородсодержащего газа на выход метанола.

объекты: малотоннажные установки по производству метанола из водородсодержащего газа, состоящие из комплекса генерации водородсодержащего газа и комплекса каталитического синтеза метанола. Комплекс генерации водородсодержащего газа включает трехкомпонентный газогенератор синтез-газа (природный газ - окислитель - химочищенная вода), в котором осуществляется парциальное окисление природного газа, блок теплообменных аппаратов и блок коррекции состава и параметров водородсодержащего газа для обеспечения отношения компонентов $\mathrm{H}_{2} / \mathrm{CO}=2,2-2,8$ и модуля $\mathrm{M}=2,0-2,3$. Комплекс каталитического синтеза метанола включает проточный каскад, состоящий из трех последовательно соединенных изотермических реакторов с выводом метанола-сырца после каждого реактора без рециркуляции отходящих и «хвостового» газов.

Методы: термодинамические расчеты.

Результаты. Подтвержден известный факт повышения удельного выхода метанола в зависимости от концентрации кислорода в окислителе на стадии парциального окисления природного газа; показано, что оптимизация состава водородсодержащего газа, идущего на каталитический синтез метанола, обеспечивает прирост удельного выхода метанола; средний удельный прирост выхода метанола при синтезе на оптимизированном составе при $M=2,05$ по сравнению с синтезом на неоптимизированном составе газа составляет 8-12%; прирост удельного выхода метанола сохраняется вне зависимости от принятой в расчетах степени конверсии газа в реакторах каскада комплекса синтеза метанола для всех типов окислителей; выявлена нелинейная зависимость удельного выхода метанола от концентрации кислорода в окислителе, заключающаяся в уменьшении прироста удельного выхода метанола при увеличении концентрации кислорода в окислителе свыше 70 \%; выявленная зависимость требует дополнительного изучения и экспериментального подтверждения, она позволяет оптимизировать эксплуатационнье затраты на окислитель за счет уменьшения удельных затрат кислорода на выход метанола из сырья.
\end{abstract}

\section{Ключевые слова:}

Метанол, синтез-газ, газогенератор, термодинамика, парциальное окисление природного газа.

\section{Введение}

Производство метанола включает стадию получения водородсодержащего газа, содержащего водород, оксиды углерода, пары воды, а также азот, остаточный метан и следовые количества других инертных газов. Термодинамические параметры газа должны обеспечивать эксплуатацию катализаторов синтеза метанола в номинальных режимах, которые для современных медьцинкалюминиевых катализаторов Великобритания - Katalko-51-9, «Johnson Matthey» (ICI), Германия - C79-7GL «Zud-Chemie» AG, Дания - МК-121 «Haldor Topsoe» - составляют по давлению 5-10 МПа, по температуре 200-280 C [1-5]. Для оптимального синтеза метанола также необходимо, чтобы модуль (факториал) водородсодержащего газа
$\mathrm{M}=\left(\mathrm{H}_{2}-\mathrm{CO}_{2}\right) /\left(\mathrm{CO}+\mathrm{CO}_{2}\right)$ находился в диапазоне $\mathrm{M}=2,0-2,3$ [2-8], что достигается при отношениях $\mathrm{H}_{2} / \mathrm{CO}=2,2-2,8$ и концентрациях $\mathrm{CO}_{2}=2-7$ \% об.

Объемный состав водородсодержащего газа зависит от способа конверсии исходного углеводородного сырья, в качестве которого преимущественно используют природный газ (ПГ). Известны традиционные способы конверсии природного газа, такие как: паровая каталитическая конверсия природного газа (ПКМ); углекислотная конверсия; парциальное окисление (ПОМ). Наиболее распространенной промышленной технологией является ПКМ, а генерируемый водородсодержащий газ обладает высокими значениями $\mathrm{H}_{2} / \mathrm{CO} \geq 4,5$ и $\mathrm{M} \geq 2,5$ [2-11]. Применение ПОМ по технологиям фирм Shell, Texaco, Lurgi позволяет полу- 
чать водородсодержащий газ с параметрами $\mathrm{H}_{2} / \mathrm{CO} \approx 1,6-1,8, \mathrm{M} \approx 1,4$ в зависимости от состава ПГ, типа окислителя (кислород, воздух или обогащенный кислородом воздух), степени увлажнения ПГ и от основного параметра режима ПОМ - коэффициента избытка окислителя [8, 12-14].

При крупнотоннажном производстве метанола и использовании в качестве исходного сырья более «жирных» газов (например, ПНГ, содержащих гомологи метана) для увеличения концентрации метана применяют предриформинг; для оптимизации тепловых балансов применяют автотермический риформинг; для оптимизации состава водородсодержащего газа, идущего на синтез метанола, применяют комбинированные технологии $[2,4,9,15]$. Так, сочетая ПКМ, ПОМ и углекислотную конверсию ПГ, оптимизируют модуль водородсодержащего газа, добиваясь повышения выхода метанола на 5-8 \% [2-4].

При малотоннажном производстве метанола, в особенности в промысловых условиях при освоении удаленных малодебитных и низконапорных месторождений, актуально применение технологии ПОМ, поскольку газогенераторы синтез-газа (ГСГ) имеют минимальные массогабаритные характеристики и позволяют создавать модульные транспортабельные установки [16-18]. Однако промышленное внедрение технологий малотоннажного производства метанола на основе ПОМ сдерживается из-за отсутствия научно-обоснованных данных о выходе метанола из сырья в зависимости от используемого типа окислителя. Также отсутствуют данные об удельных капитальных затратах и себестоимости продукции в зависимости от технических решений по оптимизации состава водородсодержащего газа, что в совокупности препятствует получению хотя бы грубой оценки маржинальности бизнеса [17-26].

В настоящей статье приводятся результаты численных экспериментов, позволяющие на примере малотоннажной установки по производству метанола с использованием технологии ПОМ оценить эффективность технических решений, связанных с оптимизацией состава водородсодержащего газа и, соответственно, увеличением степени конверсии водородсодержащего газа в метанол.

\section{Описание объекта и программы исследований}

Объектом исследования является малотоннажная установка по производству метанола [9, 18, 21-27], состоящая из ГСГ $[9,14]$, реализующего процесс некаталитического ПОМ и проточного каскада из трех изотермических реакторов синтеза метанола [10]. Также установка содержит блок коррекции состава водородсодержащего газа $[9,28]$. Упрощенная блоксхема установки представлена на рис. 1.

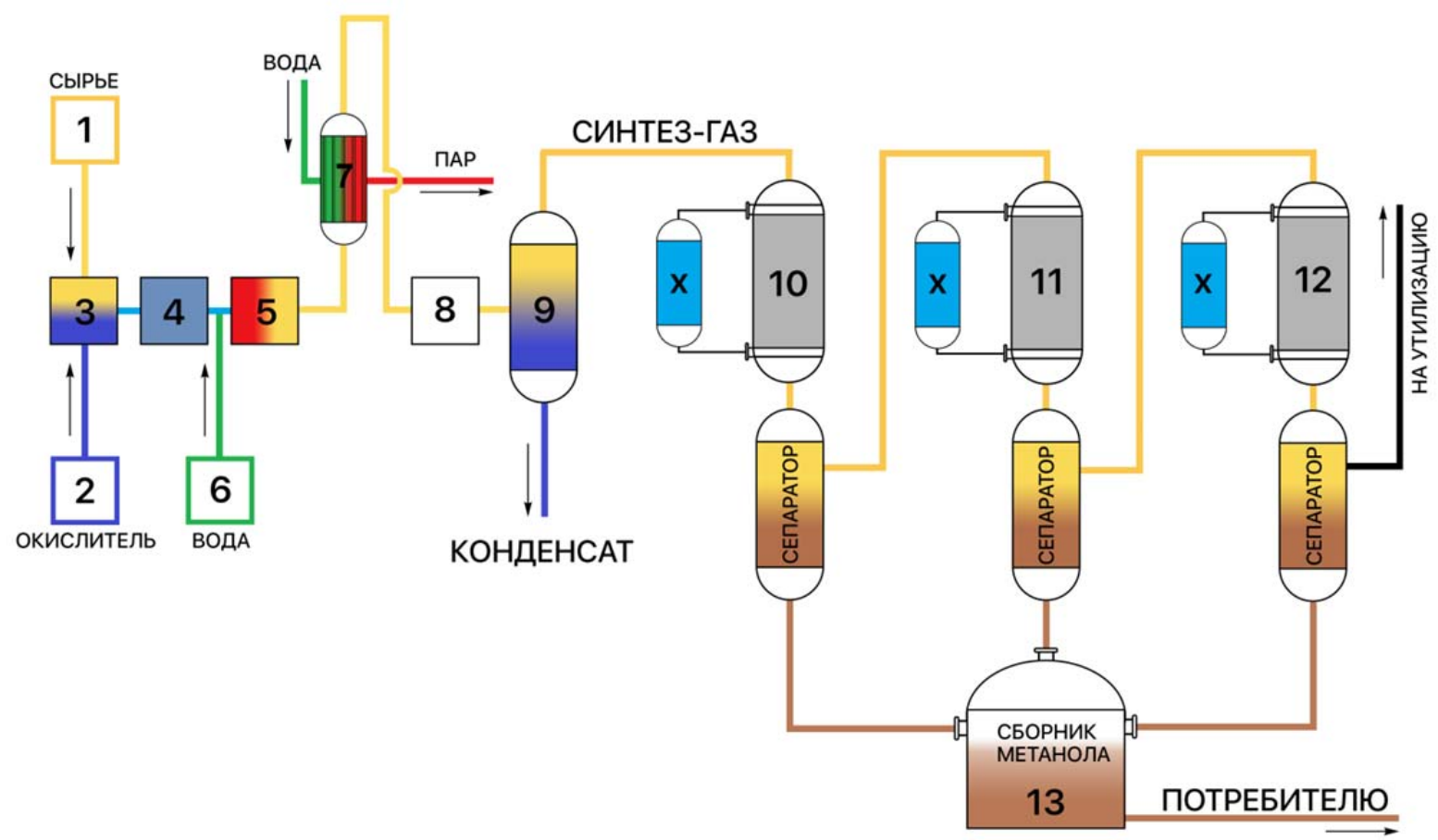

Pис. 1. Упрощенная блок-схема установки по производству метанола: 1 - узел ввода сырья, 2 - узел ввода окислителя, 3 - смесительная головка ГСГ, 4 - камера сгорания (КС) ГСГ, 5 - испарительная камера (ИК) ГСГ, 6 узел ввода химочищенный воды в ИК, 7 - котел-утилизатор, 8 - блок коррекции, 9 - сепаратор, 10-12 - реакторы синтеза метанола, 13 - накопитель метанола, $X$ - холодильник

Fig. 1. Simplified flow diagram of a methanol production plant: 1 - raw material input unit, 2 - oxidizer input unit, 3 - mixing head of a syngas generator, 4 - combustion chamber (CC) of a syngas generator, 5 - evaporation chamber (EC) of a syngas generator, 6 - chemical water input unit of a syngas generator, 7 - recovery boiler, 8 - correction unit, 9 - separator, 10-12-methanol synthesis reactors, 13 - methanol storage unit, $X$-refrigerator 
Исходным углеводородным сырьем является ПГ, имеющий следующий состав, об. \%: $\mathrm{CH}_{4}-97,57$, $\mathrm{C}_{2} \mathrm{H}_{6}-1,0, \mathrm{C}_{3} \mathrm{H}_{8}-0,37, \mathrm{C}_{4} \mathrm{H}_{10}-0,15, \mathrm{~N}_{2}-0,84, \mathrm{CO}_{2}-$ 0,07 . ПГ смешивают с водяным паром, составляющим $15 \%$ по массе относительно массы сырья, и через узел ввода - 1 подают в смесительную головку ГСГ 3. В качестве окислителя рассматривались кислород, воздух, обогащенный воздух с концентрацией $\mathrm{O}_{2}-$ $35 \%$ об., обогащенный воздух с концентрацией $\mathrm{O}_{2}-$ $70 \%$ об. Окислитель через узел ввода - 2 подают в смесительную головку ГСГ. Начальные параметры режима ПОМ: давление подачи компонентов 6,5 МПа, температура увлажненного сырья - $400{ }^{\circ} \mathrm{C}$, температура кислорода $-170{ }^{\circ} \mathrm{C}$, начальная температура остальных типов окислителей $-250{ }^{\circ} \mathrm{C}$. Значения коэффициента избытка окислителя $\alpha$ принимались в соответствии с рекомендациями работ $[14,15,20,27$, 29, 30], а именно: $\alpha=0,34$ - для компонентов ПГ кислород, $\alpha=0,35$ - для компонентов ПГ - обогащенный воздух $\left(\mathrm{O}_{2}-70 \%\right.$ об. $), \alpha=0,36$ - для компонентов ПГ - обогащенный воздух $\left(\mathrm{O}_{2}-35 \%\right.$ об.), $\alpha=0,38$ для компонентов ПГ - воздух. Конструкция смесительной головки обеспечивает турбулентный режим подачи окислителя и нагретого увлажненного ПГ в КС - 4, в которой протекает процесс некаталитического парциального окисления ПГ при давлении 6,0 МПа. Продукты ПОМ из КС поступают в ИК - 5, где охлаждаются путем впрыска химочищенной воды через узел ввода - 6. Температура газового потока на выходе ИК (выходе ГСГ) является регулируемой, в расчетах ее значение принималось $900{ }^{\circ} \mathrm{C}$. Газ с выхода ИК используют для нагрева сырья и окислителя, а также выработки водяного пара в котлеутилизаторе - 7, откуда водородсодержащий газ поступает в блок коррекции - 8 для коррекции концентраций $\mathrm{H}_{2}, \mathrm{CO}$ и $\mathrm{CO}_{2}$ и оптимизации модуля М. Газ с выхода блока коррекции охлаждают, после чего в сепараторе - 9 отделяют водяной конденсат. Отходящий из 9 газовый поток нагревают до $210{ }^{\circ} \mathrm{C}$ и подают в блок синтеза метанола под давлением 5,0 МПа. В реакторах синтеза метанола применяют катализатор C79-7GL. Газовые потоки с выхода реакторов 10-12 охлаждают, отделяют метанол-сырец и подают его в накопитель - 13, а отходящие газы нагревают и подают в следующий по ходу газового потока реактор синтеза метанола. Хвостовые газы из реактора - 12 утилизируют.

Программа численных исследований включала три этапа. Первый этап заключался в расчете термодинамики процесса парциального окисления природного газа в ГСГ для указанных сочетаний компонентов и параметров режима ПОМ с целью получения балансовых характеристик водородсодержащего газа на выходе ГСГ. Второй этап включал расчет оптимизированных балансовых составов водородсодержащего газа на входе в блок синтеза метанола. На третьем этапе проводились собственно расчеты выходов метанола в реакторах каскада синтеза метанола для различных вариантов состава газа.

\section{Описание методики и результатов}

\section{численных экспериментов}

В табл. 1-4 представлены фрагменты термодинамических расчетов парциального окисления ПГ приведенного состава. В расчетах не учитывался сопутствующий ПОМ нежелательный процесс «сажеобразования», кинетика которого недостаточно изучена [31-35]. С целью профилактики сажеобразования в установке предусмотрены специальные меры, связанные с улучшением смесеобразования, увлажнением исходного сырья и др. [22, 29]. Увлажнение сырья и впрыск воды в испарительную камеру приводят к увеличению отношения $\mathrm{H}_{2} / \mathrm{CO}$ вследствие протекания реакции водяного газа и слабой конверсии оксида углерода при отсутствии катализатора. Этот факт учитывался при моделировании путем искусственного введения 5 \%-го ограничения на степень конверсии $\mathrm{CO}$, что согласуется с экспериментальными данными работ $[16,30,32]$.

Таблица 1. Мольный состав газовых потоков в ГСГ при парииальном окислении ПГ воздухом

Table 1. Molar composition of gas flows in the syngas generator during partial oxidation of natural gas by air

\begin{tabular}{|l|c|c|c|c|c|c|c|c|c|}
\hline \multicolumn{1}{|c|}{$\begin{array}{l}\text { Поток } \\
\text { Flow }\end{array}$} & $\mathrm{T},{ }^{\circ} \mathrm{C}$ & \multicolumn{7}{|c|}{ Состав/Composition } & \multirow{2}{*}{$\mathrm{CH}_{2} / \mathrm{CO}$} \\
\hline $\begin{array}{l}\text { окисли- } \\
\text { тель в 3* } \\
\text { oxidant } \\
\text { in 3* }\end{array}$ & 250 & - & - & - & - & - & 0,78 & 0,22 & - \\
\hline $\begin{array}{l}\text { cыpье в 3* } \\
\text { feedstock } \\
\text { in 3* }\end{array}$ & 400 & 0,850 & - & - & - & 0,130 & 0,008 & - & - \\
\hline $\begin{array}{l}\text { га3 после 4* } \\
\text { gas after 4* }\end{array}$ & 1250 & 0,000 & 0,143 & 0,023 & 0,242 & 0,107 & 0,485 & - & 1,70 \\
\hline $\begin{array}{l}\text { га3 после 5* } \\
\text { gas after 5* }\end{array}$ & 900 & 0,000 & 0,118 & 0,025 & 0,213 & 0,227 & 0,417 & - & 1,81 \\
\hline
\end{tabular}

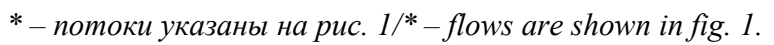

Таблица 2. Мольный состав газовых потоков в ГСГ при парииальном окислении ПГ обогащенным воздухом с конщентрацией кислорода $35 \%$ об.

Table 2. Molar composition of gas flows in the syngas generator during partial oxidation of natural gas with enriched air with an oxygen concentration of $35 \% \mathrm{vol}$.

\begin{tabular}{|c|c|c|c|c|c|c|c|c|c|}
\hline \multirow{2}{*}{$\begin{array}{c}\text { Поток } \\
\text { Flow }\end{array}$} & \multirow{2}{*}{$\mathrm{T},{ }^{\circ} \mathrm{C}$} & \multicolumn{7}{|c|}{ Состав/Composition } & \multirow{2}{*}{$\mathrm{H}_{2} / \mathrm{CO}$} \\
\hline & & $\mathrm{CH}_{4}$ & $\mathrm{CO}$ & $\mathrm{CO}_{2}$ & $\mathrm{H}_{2}$ & $\mathrm{H}_{2} \mathrm{O}$ & $\mathrm{N}_{2}$ & $\mathrm{O}_{2}$ & \\
\hline $\begin{array}{l}\text { окислитель } \\
\text { в 3* } \\
\text { oxidant } \\
\text { in 3* }\end{array}$ & 250 & - & - & - & - & - & 0,65 & 0,35 & - \\
\hline $\begin{array}{l}\text { сырье в 3* } \\
\text { feedstock } \\
\text { in 3* }\end{array}$ & 400 & 0,850 & - & - & - & 0,130 & 0,008 & - & - \\
\hline $\begin{array}{l}\text { газ после } \\
4 * \\
\text { gas after } 4 *\end{array}$ & 1430 & 0,00 & 0,196 & 0,023 & 0,329 & 0,132 & 0,320 & - & 1,68 \\
\hline $\begin{array}{l}\text { газ после } \\
5^{*} \\
\text { gas after } 5 *\end{array}$ & 900 & 0,000 & 0,214 & 0,034 & 0,384 & 0,003 & 0,365 & - & 1,79 \\
\hline
\end{tabular}


Таблица 3. Мольный состав газовых потоков в ГСГ при парииальном окислении ПГ обогащенным воздухом с концентрацией кислорода $70 \%$ об.

Table 3. Molar composition of gas flows in the syngas generator during partial oxidation of natural gas with enriched air with an oxygen concentration of $70 \%$ vol.

\begin{tabular}{|l|c|c|c|c|c|c|c|c|c|}
\hline $\begin{array}{c}\text { Поток } \\
\text { Flow }\end{array}$ & \multirow{2}{*}{${ }^{\circ} \mathrm{C}$} & \multicolumn{7}{|c|}{ Состав/Composition } & \multicolumn{2}{|c|}{$\mathrm{H}_{2} / \mathrm{CO}$} \\
\hline $\begin{array}{l}\text { окислитель } \\
\text { в 3* } \\
\text { oxidant in 3* }\end{array}$ & 250 & - & - & - & - & - & 0,30 & 0,70 & - \\
\hline $\begin{array}{l}\text { cыpье в 3* } \\
\text { feedstock } \\
\text { in 3* }\end{array}$ & 400 & 0,850 & - & - & - & 0,130 & 0,008 & - & - \\
\hline $\begin{array}{l}\text { га3 после 4* } \\
\text { gas after 4* }\end{array}$ & 1400 & 0,001 & 0,272 & 0,021 & 0,493 & 0,126 & 0,088 & - & 1,81 \\
\hline $\begin{array}{l}\text { ra3 после 5* } \\
\text { gas after 5* }\end{array}$ & 900 & 0,001 & 0,212 & 0,026 & 0,409 & 0,281 & 0,071 & - & 1,93 \\
\hline
\end{tabular}

*-потоки указаны на рис. $1 /{ }^{*}$-flows are shown in fig. 1 .

Таблица 4. Мольный состав газовых потоков в ГСГ при парииальном окислении ПГ кислородом

Table 4. Molar composition of gas flows in the syngas generator during partial oxidation of natural gas with oxygen

\begin{tabular}{|l|c|c|c|c|c|c|c|c|c|}
\hline $\begin{array}{c}\text { Поток } \\
\text { Flow }\end{array}$ & \multirow{2}{*}{${ }^{\circ} \mathrm{C}$} & \multicolumn{7}{|c|}{ Состав/Composition } & \multicolumn{2}{|c|}{$\mathrm{H}_{2} / \mathrm{CO}$} \\
\hline $\begin{array}{l}\text { окислитель } \\
\text { в 3* } \\
\text { oxidant in 3* }\end{array}$ & 170 & - & - & - & - & - & - & 1,0 & - \\
\hline $\begin{array}{l}\text { cыpье в 3* } \\
\text { feedstock } \\
\text { in 3* }\end{array}$ & 400 & 0,850 & - & - & - & 0,130 & 0,008 & - & - \\
\hline $\begin{array}{l}\text { газ после 4* } \\
\text { gas after 4* }\end{array}$ & 1490 & 0,003 & 0,300 & 0,021 & 0,536 & 0,141 & 0,003 & - & 1,79 \\
\hline $\begin{array}{l}\text { га3 после 5* } \\
\text { gas after 5* }\end{array}$ & 900 & 0,000 & 0,225 & 0,026 & 0,429 & 0,317 & 0,002 & - & 1,91 \\
\hline
\end{tabular}

*-nотоки указаны на рис. $1 /{ }^{*}-$ flows are shown in fig. 1 .

Полученные данные позволяют сформулировать следующие выводы:

- $\mathrm{H}_{2} / \mathrm{CO}$ в газе на выходе камеры сгорания ГСГ изменяется в диапазоне от 1,6 до 1,8 , что в определяющей степени зависит от значений коэффициента избытка окислителя;

- при отсутствии коррекции состава водородсодержащего газа $\mathrm{M}<1,5$ при любых вариациях параметров режима ПОМ и типа окислителя.

Таким образом, показатели качества состава водородсодержащего газа, получаемого в результате ПОМ, для любых типов окислителей существенно ниже рекомендуемых значений для оптимального синтеза метанола.

С целью оптимизации состава водородсодержащего газа предложены способы, устройства и алгоритмы [28-30]. В соответствии с рекомендациями этих работ выполнены расчеты скорректированных материальных балансов газовых потоков для всех вариантов составов, приведенных в табл. 1-4. Оптимизированные и неоптимизированные варианты составов газовых потоков с учетом теплообменных процессов и сепарации водяного пара перед подачей газа в комплекс синтеза метанола являются исходными данными для расчетов выхода метанола в реакторах 11-13.
Для расчета процесса конверсии могут быть использованы два известных в литературе механизма каталитического синтеза метанола и их комбинация. Первый описывается двумя экзотермическими макрореакциями в присутствии цинк-хромовых катализаторов [2, 3]: $\mathrm{CO}+2 \mathrm{H}_{2}=\mathrm{CH}_{3} \mathrm{OH}, \quad \mathrm{CO}_{2}+\mathrm{H}_{2}=\mathrm{CO}+\mathrm{H}_{2} \mathrm{O}$. Второй механизм предложен А.Я. Розовским применительно к каталитическому синтезу метанола на медьсодержащих катализаторах и также основан на протекании двух экзотермических макрореакций [3]: $\mathrm{CO}_{2}+3 \mathrm{H}_{2}=\mathrm{CH}_{3} \mathrm{OH}+\mathrm{H}_{2} \mathrm{O}, \mathrm{CO}+\mathrm{H}_{2} \mathrm{O}=\mathrm{CO}_{2}+\mathrm{H}_{2}$.

Все приведенные реакции являются обратимыми. Основными факторами, влияющими на выход метанола, скорость протекания реакций и степень конверсии оксидов углерода, являются: давление, температура в зоне реакции, отношения $\mathrm{H}_{2} / \mathrm{CO}$ и $\mathrm{CO} / \mathrm{CO}_{2}$, значение модуля газа, содержание водяного пара и инертных компонентов в реакционной зоне [1-4, 17-19]. Наличие инертных компонентов - остаточного метана и азота - снижает парциальное (эффективное) давление реагирующих веществ, вследствие чего степень конверсии оксидов углерода уменьшается [2-4, 17-19]. Основным ингибитором синтеза является водяной пар $[2,3]$. По указанной причине для увеличения степени конверсии водородсодержащего газа в описываемой установке применяют проточный трехреакторный каскад с выводом продуктов - метанола и воды после каждого реактора $[3,19]$.

По мнению многих авторов, на практике работают оба механизма синтеза метанола через оксиды углерода. Расчеты равновесия по обоим механизмам дают близкие результаты и хорошее совпадение с экспериментальными данными $[4,20]$. Проведено моделирование равновесных процессов выхода метанола для различных вариантов балансовых составов водородсодержащего газа, поступающих в первый реактор 10 каскада синтеза метанола. Варианты отличаются используемым типом окислителя (воздух, обогащенный воздух, кислород), а также наличием или отсутствием коррекции состава. В табл. 5, 6 представлены фрагменты результатов численных расчетов для компонентов ПГ - обогащенный воздух с концентрацией кислорода 70 \% об. Объемная скорость катализа принималась равной 10000, номинальный массовый расход газа на входе в первый реактор каскада составлял 1000-3400 кг/ч в зависимости от концентрации кислорода в окислителе, что соответствует расчетной производительности установки 2500-5000 т метанола в год [36].

Итоговые результаты моделирования процессов выхода метанола (количество метанола в кг, получаемое из 1000 нм $^{3}$ природного газа) в трехреакторном каскаде синтеза метанола для разных вариантов исходных данных, отличающихся концентрацией кислорода в окислителе, степенью конверсии синтез-газа в каждом реакторе каскада, наличием или отсутствием коррекции состава синтез-газа, отображены на рис. 2. В табл. 7 представлены материальные балансы в малотоннажной установке при различных принимаемых в расчетах степенях конверсии газа, убывающих в каждом последующем реакторе синтеза (50 \%-20 \%-10 \%). 
Таблица 5. Материальный баланс комплекса синтеза метанола (окислитель - обогащенный воздух, $\mathrm{O}_{2}-70$ \% об., $M=1,62)$

Table 5. Material balance of the methanol synthesis complex (oxidizer-enriched air, $\mathrm{O}_{2}-70 \%$ vol., $M=1,62$ )

\begin{tabular}{|c|c|c|c|c|c|c|c|c|c|}
\hline \multirow{2}{*}{$\begin{array}{l}\text { Поток } \\
\text { Flow }\end{array}$} & \multirow{2}{*}{$\mathrm{T},{ }^{\circ} \mathrm{C}$} & \multirow{2}{*}{$\begin{array}{c}\text { Расход/Consumption } \\
\kappa г / ч\end{array}$} & \multicolumn{7}{|c|}{ Состав/Composition } \\
\hline & & & $\mathrm{CH}_{4}$ & $\mathrm{CO}$ & $\mathrm{CO}_{2}$ & $\mathrm{H}_{2}$ & $\mathrm{H}_{2} \mathrm{O}$ & $\mathrm{N}_{2}$ & $\mathrm{MeOH}$ \\
\hline $\begin{array}{l}\text { газ в реактор } 10^{*} \\
\text { gas to reactor } 10^{*}\end{array}$ & 210 & 1265 & 0,001 & 0,243 & 0,073 & 0,585 & 0,003 & 0,095 & 0,000 \\
\hline $\begin{array}{l}\text { метанол из } 10^{*} \\
\text { methanol out of } 10^{*}\end{array}$ & 40 & 360 & 0,000 & 0,002 & 0,036 & 0,003 & 0,024 & 0,003 & 0,935 \\
\hline $\begin{array}{l}\text { газ в реактор } 11^{*} \\
\text { gas to reactor } 11^{*}\end{array}$ & 220 & 905 & 0,002 & 0,192 & 0,109 & 0,539 & 0,000 & 0,149 & 0,009 \\
\hline $\begin{array}{l}\text { метанол из } 11^{*} \\
\text { methanol out of } 11 *\end{array}$ & 40 & 76 & 0,000 & 0,002 & 0,037 & 0,004 & 0,001 & 0,004 & 0,953 \\
\hline $\begin{array}{l}\text { газ в реактор } 12 * \\
\text { gas to reactor } 12 *\end{array}$ & 230 & 829 & 0,002 & 0,174 & 0,122 & 0,524 & 0,000 & 0,169 & 0,009 \\
\hline $\begin{array}{l}\text { метанол из } 12 * \\
\text { methanol out of } 12 *\end{array}$ & 40 & 31 & 0,000 & 0,002 & 0,039 & 0,004 & 0,000 & 0,004 & 0,952 \\
\hline $\begin{array}{l}\text { хвостовые газы } \\
\text { (на утилизацию) } \\
\text { tail gases (for recycling) }\end{array}$ & 40 & 798 & 0,002 & 0,165 & 0,128 & 0,517 & 0,000 & 0,179 & 0,009 \\
\hline
\end{tabular}

*- потоки указаны на рис. $1 /{ }^{*}$-flows are shown in fig. 1 .

Таблица 6. Материальный баланс комплекса синтеза метанола (окислитель - обогащенный воздух, $\mathrm{O}_{2}-70 \%$ об., $M=2,05)$

Table 6. Material balance of the methanol synthesis complex (oxidizer-enriched air, $\mathrm{O}_{2}-70 \%$ vol., $M=2,05$ )

\begin{tabular}{|c|c|c|c|c|c|c|c|c|c|}
\hline \multirow{2}{*}{$\begin{array}{l}\text { Поток } \\
\text { Flow }\end{array}$} & \multirow{2}{*}{$\mathrm{T},{ }^{\circ} \mathrm{C}$} & \multirow{2}{*}{$\begin{array}{c}\text { Расход/Consumption } \\
\kappa г / ч\end{array}$} & \multicolumn{7}{|c|}{ Состав/Composition } \\
\hline & & & $\mathrm{CH}_{4}$ & $\mathrm{CO}$ & $\mathrm{CO}_{2}$ & $\mathrm{H}_{2}$ & $\mathrm{H}_{2} \mathrm{O}$ & $\mathrm{N}_{2}$ & $\mathrm{MeOH}$ \\
\hline $\begin{array}{l}\text { газ в реактор } 10^{*} \\
\text { gas to reactor } 10^{*}\end{array}$ & 210 & 1200 & 0,001 & 0,255 & 0,029 & 0,612 & 0,003 & 0,100 & 0,000 \\
\hline $\begin{array}{l}\text { метанол из } 10^{*} \\
\text { methanol out of } 10^{*}\end{array}$ & 40 & 389 & 0,000 & 0,002 & 0,013 & 0,004 & 0,004 & 0,004 & 0,954 \\
\hline $\begin{array}{l}\text { газ в реактор } 11^{*} \\
\text { gas to reactor } 11^{*}\end{array}$ & 220 & 811 & 0,002 & 0,206 & 0,044 & 0,578 & 0,000 & 0,161 & 0,009 \\
\hline $\begin{array}{l}\text { метанол из } 11^{*} \\
\text { methanol out of } 11 *\end{array}$ & 40 & 82 & 0,000 & 0,002 & 0,015 & 0,004 & 0,000 & 0,004 & 0,974 \\
\hline $\begin{array}{l}\text { газ в реактор } 12 * \\
\text { gas to reactor } 12 *\end{array}$ & 230 & 729 & 0,002 & 0,189 & 0,050 & 0,566 & 0,000 & 0,184 & 0,009 \\
\hline $\begin{array}{l}\text { метанол из } 12 * \\
\text { methanol out of } 12 *\end{array}$ & 40 & 33 & 0,000 & 0,002 & 0,016 & 0,004 & 0,000 & 0,005 & 0,974 \\
\hline $\begin{array}{l}\text { хвостовые газы } \\
\text { (на утилизацию) } \\
\text { tail gases } \\
\text { (for recycling) }\end{array}$ & 40 & 696 & 0,002 & 0,180 & 0,053 & 0,561 & 0,000 & 0,195 & 0,009 \\
\hline
\end{tabular}

*- потоки указаны на рис. $1 / *$-flows are shown in fig. 1 .

Таблица 7. Сводные балансовые расходы в установке

Table 7. Summary balance sheet expenses in the installation

\begin{tabular}{|c|c|c|c|c|}
\hline$\underbrace{\begin{array}{c}\text { Расходы } \\
\text { Consumption }\end{array}}_{\substack{\text { Концентрация } \\
\mathrm{O}_{2}, \% \text { об. }}}$ & $\begin{array}{c}\text { Поток газа в газоге- } \\
\text { нераторе } \\
\text { Gas consumption in } \\
\text { the syngas generator }\end{array}$ & $\begin{array}{c}\text { Поток газа в блоке синтеза } \\
\text { метанола } \\
\text { Gas consumption in the methanol } \\
\text { synthesis unit }\end{array}$ & $\begin{array}{c}\text { Выход } \\
\text { метанола } \\
\text { Methanol yield }\end{array}$ & \multirow[t]{2}{*}{$\begin{array}{c}\text { Мощность } \\
\text { установки, } \\
\text { т/год } \\
\text { Plant capacity, } \\
\text { t/year } \\
\end{array}$} \\
\hline Density, $\mathrm{O}_{2}, \%$ vol. & \multicolumn{3}{|c|}{$\mathrm{\kappa г} / \mathrm{\varphi} / \mathrm{kg} /$ hour } & \\
\hline $\begin{array}{l}22 \% \text { б/K* } \\
\mathrm{w} / \mathrm{c}\end{array}$ & 3650 & 3431 & 315 & 2759 \\
\hline $\begin{array}{l}22 \% \mathrm{c} / \mathrm{K}^{* *} \\
\mathrm{a} / \mathrm{c}\end{array}$ & 3650 & 3341 & 363 & 3180 \\
\hline $\begin{array}{l}35 \% \text { б/к } \\
\mathrm{w} / \mathrm{c}\end{array}$ & 2438 & 2235 & 363 & 3180 \\
\hline $\begin{array}{l}35 \% \mathrm{c} / \mathrm{K} \\
\mathrm{a} / \mathrm{c}\end{array}$ & 2438 & 2149 & 416 & 3644 \\
\hline $\begin{array}{l}70 \% \text { б/к } \\
\mathrm{w} / \mathrm{c}\end{array}$ & 1399 & 1265 & 437 & 3828 \\
\hline $\begin{array}{l}70 \% \mathrm{c} / \mathrm{K} \\
\mathrm{a} / \mathrm{c}\end{array}$ & 1399 & 1201 & 486 & 4257 \\
\hline $\begin{array}{l}100 \% \text { б/к } \\
\mathrm{w} / \mathrm{c}\end{array}$ & 1168 & 1016 & 449 & 3933 \\
\hline $\begin{array}{l}100 \% \mathrm{c} / \mathrm{K} \\
\mathrm{a} / \mathrm{c}\end{array}$ & 1168 & 962 & 526 & 4607 \\
\hline
\end{tabular}

*б/к - без коррекиии состава газа, **/к- после коррекиии состава газа.

$* w / c-$ without correction gas composition, ${ }^{* *}$ a/c - after correction of gas composition. 


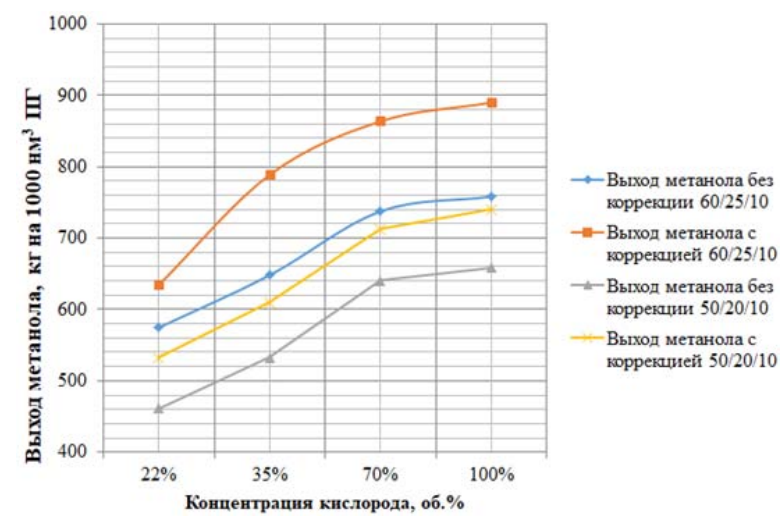

Рис. 2. Зависимость выхода метанола от типа окислителя, параметров синтез-газа и степени конверсии синтез-газа в реакторах каскада синтеза метанола

Fig. 2. Dependence of methanol yield on the oxidizer type, synthesis gas parameters, and synthesis gas conversion degree in methanol synthesis cascade reactors

\section{Заключение}

Во-первых, полученные результаты подтверждают известный факт, что повышение выхода метанола достигается, прежде всего, увеличением концентрации кислорода в окислителе при проведении парциального окисления. Так, при концентрации кислорода

\section{СПИСОК ЛИТЕРАТУРЫ}

1. Wernicke H.-J., Plass L., Schmidt F. Methanol generation Methanol: the basic chemical and energy feedstock of the future. Berlin: Springer-Verlag Berlin Heidelberg, 2014. - P. 51-301.

2. Технология синтетического метанола / М.М. Караваев, В.Е. Леонов, И.Г. Попов, Е.Т. Шепелев. - М.: Изд-во: «Химия», 1984. - 240 с.

3. Розовский А.Я., Лин Г.И. Теоретические основы процесса синтеза метанола. - М.: Изд-во: «Химия», 1990. - 272 с.

4. Технология автотермического риформинга для современных крупнотоннажных метанольных установок / П.Ю. Даль, Т.С. Кристенсен, С. Винтер-Мадсен, С.М. Кинг // Азот и синтез-газ-2014: Международная конференция. - Париж: Хальдор Tопсе A/O, 2014. - 14 c.

5. Westerterp K.R. New methanol processes // Pilavachi P.A. Energy efficiency in process technology. - Dordrecht: Springer, 1993 P. 1142-1153.

6. Liu W.-C., Baek J., Somorjai G.A. The Methanol economy: methane and carbon dioxide conversion // Topics in Catalysis. 2018. - Iss. 7-8. - P. 530-541.

7. Glazov S.V. Estimation of the characteristics of the partial oxidation conversion of organic fuel gasification products to a synthesis gas, Russia, Moscow region // Theoretical Foundations of Chemical Engineering. - 2019. - V. 53. - № 3. - P. 378-388.

8. Способ синтеза метанола: пат. Рос. Федерации, № 2519940 , заявл. 20.04.2013; опубл. 20.06.2014. Бюл. № 11. - 14 с.

9. Способ получения водородсодержащего газа для производства метанола и устройство для его осуществления пат. Рос Федерации, № 2632846, заявл. 15.08.2016; опубл. 10.10.2017. Бюл. № 28. -4 c.

10. Малотоннажная установка получения метанола пат. Рос. Федерации, № 176510, заявл. 20.06.2017; опубл. 22.01.2018. Бюл. № 3. $-3 \mathrm{c}$.

11. Загашвили Ю.В., Левихин А.А., Кузьмин А.М. Основы проектирования трехкомпонентного газогенератора синтез-газа // Нефтегазохимия. - 2017. - № 4. - С. 9-16.

12. Experimental study and kinetics modeling of partial oxidation reactions in heavily sooting laminar premixed methane flames, China, Beijing / Q. Li, T. Wang, Y. Liu, D. Wang, // Chemical Engineering Journal. - 2012. - V. 207-208. - № 1. - P. 235-244. в окислителе 35 \% выход метанола по сравнению с воздухом в качестве окислителя увеличивается на $15 \%$, при $70 \%$ - на $39 \%$, при использовании технически чистого кислорода - на 43 \%. Этот вывод качественно и количественно подтверждает известные результаты работ $[19,20]$, в которых рассмотрены особенности синтеза метанола на забалластированном азотом синтез-газе.

Во-вторых, коррекция состава водородсодержащего газа обеспечивает дополнительный прирост выхода метанола на 8-12\%, причем относительный прирост остается примерно постоянным вне зависимости от принимаемой в расчетах степени конверсии газа в реакторах каскада синтеза метанола. Однако количественная оценка увеличения выхода метанола нуждается в экспериментальной проверке.

В-третьих, наблюдается нелинейная зависимость удельного выхода метанола от концентрации кислорода в окислителе, проявляющаяся в относительном уменьшении приростов выхода метанола с увеличением концентрации кислорода, в особенности в диапазоне концентраций свыше 70 \% об. Найденная закономерность требует дополнительного изучения, в случае ее экспериментального подтверждения возможна оптимизация эксплуатационных расходов на окислитель.

13. Influence of temperature and pressure on the non-catalytic partial oxidation of natural gas / P. Brüggemann, P. Seifert, B. Meyer, M. Müller-Hagedorn // Chemical Product and Process Modeling. 2010. - V. 5. - № 1. - P. 1-24.

14. Process analysis of syngas production by non-catalytic POX of oven gas, China, Shanghai / F. Wang, X. Zhou, W. Guo, Z. Dai, X. Gong, H. Liu, Y. Guangsuo, Y. Zunhong // Frontiers of Energy and Power Engineering in China. - 2008. - V. 5. - № 1. - P. 117-122.

15. Загашвили Ю.В., Левихин А.А., Кузьмин А.М. Технология получения водорода с использованием малогабаритных транспортабельных установок на основе высокотемпературных газогенераторов синтез-газа // Вопросы материаловедения. - 2017. - № 2. - С. 92-109.

16. Управление технологическим процессом получения синтез-газа в высокотемпературном реакторе / Ю.В. Загашвили, Ю.В. Анискевич, А.М. Кузьмин, А.А. Левихин, Г.Б. Савченко // Мехатроника, автоматизация, управление. - 2015. - Т. 16. № 10. - С. 704-709.

17. Метанол 2019. URL: http://www.creon-conferences.com/ consulting/detailConf.php?ID=126542 (дата обращения 15.03.2020).

18. Synthesizing methanol from nitrogen-ballasted syngas, Russia, Moscow I.I. Lishchiner, O.V. Malova, A.L. Tarasov, V.M. Maslennikov, Y.A. Vyskubenko, L.S. Tolchinskii, Y.L. Dolinskii // Catalysis in Industry. - 2010. - V. 2. - № 4. - P. 368-373.

19. Лапидус А.Л., Елисеев О.Л., Крючков М.В. Получение углеводородов из синтез-газа, забалластированного азотом // Технология нефти и газа. - 2011. - № 5. - С. 9-12.

20. Арутюнов В.С., Савченко В.И., Седов И.В. О перспективах промысловых газохимических технологий на основе азотсодержащего синтез-газа // Нефтегазохимия. - 2016. - № 4. C. $14-23$.

21. Комплекс получения синтез-газа для малотоннажного производства метанола / Ю.В. Загашвили, В.Н. Ефремов, А.М. Кузьмин, И.И. Лищинер // Нефтегазохимия. - 2017. - № 1. - С. 19-26.

22. Bockhorn H. Soot formation in combustion. - Germany: SpringerVerlag, 1994. - 596 p.

23. Zhou X., Chen C., Wang F. Multi-dimensional modeling of noncatalytic partial oxidation of natural gas in a high pressure reformer, China, Shanghai // Journal Hydrogen Energy. - 2010. V. 35. - № 4. - P. 1620-1629. 
24. Jaber O., Naterer G.F., Dincer I. Natural gas usage as a heat source for integrated SMR and thermochemical hydrogen production technologies, Canada, Ontario // International Journal of Hydrogen Energy. - 2010. - V. 35. - P. 8569-8579.

25. Numerical simulation of natural gas non-catalytic partial oxidation reformer, China, Shanghai / Y. Xu, Z. Dai, C. Li, X. Li, Z. Zhou, G. Yu, F. Wang // International Journal Hydrogen Energy. 2014. - V. 39. - P. 9149-9157.

26. Mevawala C., Jiang Y., Bhattacharyya D. Techno-economic optimization of shale gas to dimethyl ether production processes via direct and indirect synthesis routes, USA, Richland // Applied Energy. - 2019. - V. 238. - P. 119-134.

27. Thermodynamic and economic analysis of a plant for the $\mathrm{CO}_{2}$ hydrogenation for methanol production, Germany, Essen $/$ D. Bellotti, M. Dierks, F. Moellenbruck, L. Magistri, K. Görner, G. Oeljeklaus // E3S Web of Conferences. - 2019. - V. 1. - P. 1-7.

28. Загашвили Ю.В., Левихин А.А., Кузьмин А.М. Основы проектирования трехкомпонентного газогенератора синтез-газа // Нефтегазохимия. - 2017. - № 4. - С. 9-16.

29. Способ получения водородсодержащего газа для производства метанола и устройство для его осуществления пат. Рос. Федерации, № 2632846, заявл. 15.08.2016; опубл. 10.10.2017. Бюл. № 28. - 4 с

30. Малотоннажная установка получения метанола пат. Рос. Федерации, № 176510, заявл. 20.06.2017; опубл. 22.01.2018. Бюл. № 3. -3 c.

31. Загашвили Ю.В., Кузьмин А.М. Управление составом синтезгаза для малотоннажного производства метанола // Технологии нефти и газа. - 2018. - № 3. - С. 54-59.
32. Образование высокодисперсной сажи при получении синтезгаза в условиях горения метана / И.В. Билера, А.А. Борисов, А.Б. Борунов, Ю.А. Колбановский, Ю.М. Королев, И.В. Россихин, И.В. Трошин // Газохимия. -2010 . - № 3. - С. 72-78.

33. Сажеобразование в процессе парциального окисления метана в условиях частичного насыщения парами воды / Д.С. Лугвищук, П.И. Кульчаковский, Э.И. Митберг, В.З. Мордкович // Нефтехимия. - 2018. - Т. 58. - № 3. - С. 324-331.

34. Arutyunov V.S., Strekova L.N. Kinetic features and technological perspectives of the partial oxidation of light alkanes, Russia, Moscow // Russian Journal of Physical Chemistry. - 2012. V. 6 (4). - P. 486-497.

35. Tran L.S., Glaude P.A., Battin-Leclerc F. Experimental study of the structure of laminar premixed flames of ethanol/methane/oxygen/argon // Combustion, Explosion, and Shock Waves. - 2013. - V. 49 (1). - P. 11-18.

36. Отчет о НИР: Теоретическое исследование режимных параметров и облика нового генератора синтез-газа, разработка автоматизированной системы контроля и управления и конструкторской документации генератора / рук. НИР А.М. Кузьмин // ООО «ГСГ». - СПб.: 2016. - 115 с. URL: http://www.rosrid.ru/nioktr/JDBZNL5ANBV1BU08S31MDOVM (дата обращения 28.03.2020).

Поступила 28.03.2020 2.

\section{Информация об авторах}

Загашвили Ю.В., доктор технических наук, профессор, генеральный директор ООО «ВТР».

Кузьммин A.M., кандидат технических наук, доцент, доцент кафедры двигателей и энергоустановок летательных аппаратов, Балтийский государственный технический университет «BOEHМЕХ» им. Д.Ф. Устинова; генеральный директор ООО «Генератор синтез-газа»; советник Российской Академии Естественных Наук. 
UDC 661.721 .422

\title{
INFLUENCE OF HYDROGEN-CONTAINING GAS COMPOSITION ON METHANOL YIELD
}

\author{
Yuriy V. Zagashvili', \\ y.zagashvili@yandex.ru
}

\author{
Aleksey M. Kuzmin 2,3,4, \\ kuzmin.lex@gmail.com \\ 1 LLC «HTR», \\ 15/1, Olgi Forsh street, Saint-Petersburg, 195297, Russia. \\ 2 Baltic State Technical University, \\ 1/21, 1st Krasnoarmeyskaya street, Saint-Petersburg, 190005, Russia. \\ 3 LLC «GSG», \\ 15/1, Olgi Forsh street, Saint-Petersburg, 195297, Russia. \\ 4 Russian Academy of Natural Sciences, \\ 38, Serpukhovskaya street, Saint-Petersburg, 190013, Russia.
}

The research relevance is caused by the lack of scientific evidence about methanol yield from natural gas depending on the type of oxidant (oxygen, enriched air or air) and optimization of structure and parameters of hydrogen-containing gas according to the criteria of the ratio of the components of the synthesis gas $\mathrm{H}_{2} / \mathrm{CO}$ and module (factorial) hydrogen-containing gas $\mathrm{M}$ for optimal methanol synthesis. The problem is particularly important for low-tonnage plants producing methanol in field conditions operating on nitrogen-ballasted hydrogencontaining gas.

The aim of the research is to evaluate the effect of optimizing the composition of hydrogen-containing gas on the yield of methanol.

Objects: low-tonnage plants for production of methanol from hydrogen-containing gas, consisting of a complex for generating hydrogencontaining gas and a complex for the catalytic synthesis of methanol. The complex for generating hydrogen-containing gas includes a three-component synthesis gas generator (natural gas - oxidizer -chemical-treated water), in which the partial oxidation of raw material is carried out, a block of heat exchangers and a block for correcting the composition and parameters of hydrogen-containing gas to ensure the ratio of components $\mathrm{H}_{2} / \mathrm{CO}=2,2-2,8$ and the module $\mathrm{M}=2,0-2,3$. The complex of catalytic synthesis of methanol includes a flow cascade consisting of three sequentially connected isothermal reactors with the output of raw methanol after each reactor without recirculating the waste and tail gases.

Methods: the thermodynamic calculations.

Results. The paper confirms the known fact of increasing the specific yield of methanol depending on the concentration of $\mathrm{O}_{2}$ in the oxidizing agent under partial oxidation of natural gas. It is shown that optimization of the hydrogen-containing gas which goes through the catalytic methanol synthesis provides an increase in specific yield of methanol. Average specific growth yield of methanol during the synthesis in an optimized composition at $M=2,05$ compared to non-optimized synthesis gas composition is $8-12 \%$. The increase in specific yield of methanol is maintained regardless of the adopted in the calculation of gas conversion degree of in the reactors of the cascade complex for the synthesis of methanol for all types of oxidizing agents. The authors have identified the nonlinear dependence of methanol yield on the concentration of $\mathrm{O}_{2}$ in the oxidant. It consists of reducing the increase in specific yield of methanol, when the concentration of $\mathrm{O}_{2}$ in the oxidizer is over $70 \%$. The revealed dependence requires additional study and experimental confirmation. It allows optimizing the operating costs of the oxidizer by reducing the specific cost of $\mathrm{O}_{2}$ on methanol yield from the raw material.

\section{Key words:}

Methanol, synthesis gas, gas generator, thermodynamics, partial oxidation of natural gas.

\section{REFERENCES}

1. Wernicke H.-J., Plass L., Schmidt F. Methanol generation. methanol: the basic chemical and energy feedstock of the future. Berlin, Springer-Verlag Berlin Heidelberg, 2014. pp. 51-301.

2. Karavayev M.M., Leonov V.E., Popov I.G., Shepelev E.T. Tekhnologiya sinteticheskogo metanola [Synthetic methanol technology]. Moscow, Khimiya Publ., 1984. 240 p.

3. Rozovskiy A.Ya., Lin G.I. Teoreticheskie osnovy protsessa sinteza metanola [The theoretical basis of the process of methanol synthesis]. Moscow, Khimiya Publ., 1990. 272 p.

4. Dal P.Yu., Kristensen T.S., Vinter-Madsen S., King S.M. Tekhnologiya avtotermicheskogo riforminga dlya sovremennykh krupnotonnazhnykh metanolnykh ustanovok [The autothermal reforming technology for modern large-scale methanol plants]. Trudy Mezhdunarodnoy konferentsii. Azot i sintez-gaz-2014 [Proc. International conference. Nitrogen and synthesis gas-2014]. Paris, 2014. $14 \mathrm{p}$.

5. Westerterp K.R. New methanol processes. Pilavachi P.A. energy efficiency in process technology. Dordrecht, Springer, 1993. pp. 1142-1153.
6. Liu W.-C., Baek J., Somorjai G.A. The Methanol economy: methane and carbon dioxide conversion. Topics in Catalysis, 2018, Iss. 7-8, pp. 530-541.

7. Glazov S.V. Estimation of the characteristics of the partial oxidation conversion of organic fuel gasification products to a synthesis gas, Russia, Moscow region. Theoretical Foundations of Chemical Engineering, 2019, vol. 53, no. 3, pp. 378-388.

8. Ostuni R., Filippi E. Sposob sinteza metanola [Method for methanol synthesis]. Patent RF, no. 2519940, 2014.

9. Zagashvili Yu.V., Efremov V.N., Kuzmin A.M. Sposob polucheniya vodorodsoderzhashchego gaza dlya proizvodstva metanola i ustroystvo dlya ego osushchestvleniya [Method of producing a hydrogen-containing gas for producing methanol and device for its implementation]. Patent RF, no. 2632846, 2017.

10. Zagashvili Yu.V., Efremov V.N., Kuzmin A.M. Malotonnazhnaya ustanovka polucheniya metanola [Low-tonnage methanol production unit]. Patent RF, no. 176510, 2018.

11. Zagashvili Yu.V., Levikhin A.A., Kuzmin A.M. Design basics for a threecomponent syngas gas generator. Neftegazokhimiya, 2017, no. 4, pp. 9-16. In Rus. 
12. Li Q., Wang T., Liu Y., Wang D. Experimental study and kinetics modeling of partial oxidation reactions in heavily sooting laminar premixed methane flames, China, Beijing. Chemical Engineering Journal, 2012, vol. 207-208, no. 1, pp. 235-244.

13. Brüggemann P., Seifert P., Meyer B., Müller-Hagedorn M. Influence of temperature and pressure on the non-catalytic partial oxidation of natural gas. Chemical Product and Process Modeling, 2010, vol. 5, pp. 1-24.

14. Wang F., Zhou X., Guo W., Dai Z., Gong X., Liu H., Guangsuo Y., Zunhong Y. Process analysis of syngas production by noncatalytic POX of oven gas, China, Shanghai. Frontiers of Energy and Power Engineering in China, 2008, vol. 5, no. 1, pp. 117-122.

15. Zagashvili Y.V., Levihin A.A., Kuzmin A.M., Production of hydrogen by using small-scale transportable plants based on hightemperature syngas generators. Voprosy Materialovedeniya, 2017 , no. 2, pp. 92-109. In Rus.

16. Zagashvili Y.V., Aniskevich Y.V., Kuzmin A.M., Levikhin A.A., Savchenko G.B. Management of technological process of producing synthesis gas in a high temperature reactor. Mekhatronika avtomatizatsiya, upravlenie, 2015, vol. 16, no. 10, pp. 704-709. In Rus.

17. Metanol 2019 [Methanol 2019]. Available at: http://www.creonconferences.com/consulting/detailConf.php?ID=126542 (accessed 15 March 2020).

18. Lishchiner I.I., Malova O.V., Tarasov A.L., Maslennikov V.M., Vyskubenko Y.A., Tolchinskii L.S., Dolinskii Y.L. Synthesizing methanol from nitrogen-ballasted syngas, Russia, Moscow. Catalysis in Industry, 2010, vol. 2, no. 4, pp. 368-373.

19. Lapidus A.L., Eliseev O.L., Kryuchkov M.V. Hydrocarbons production from synthesis gas, ballasted with nitrogen. Science and Technology of Hydrocarbons, 2011, no. 5, pp. 9-12.

20. Arutyunov V.S., Savchenko V.I., Sedov I.V., On the prospects of commercial gas chemical technologies based on nitrogencontaining synthesis gas. NefteGazoKhimiya, 2016, no. 4, pp. 14-23. In Rus.

21. Zagashvili Yu.V., Efremov V.N., Kuzmin A.M., Lishchiner I.I. Complex for obtaining synthesisgas for small tonnage production of methanol. Neftegazokhimiya, 2017, no. 1, pp. 19-26. In Rus.

22. Bockhorn H. Soot formation in combustion. Germany, SpringerVerlag, 1994. $596 \mathrm{p}$

23. Zhou X., Chen C., Wang F. Multi-dimensional modeling of noncatalytic partial oxidation of natural gas in a high pressure reformer, China, Shanghai. Journal Hydrogen Energy, 2010, vol. 35, no. 4, pp. $1620-1629$.

24. Jaber O., Naterer G.F., Dincer I. Natural gas usage as a heat source for integrated SMR and thermochemical hydrogen production technologies, Canada, Ontario. International Journal of Hydrogen Energy, 2010, vol. 35, pp. 8569-8579.

25. Xu Y., Dai Z., Li C., Li X., Zhou Z., Yu G., Wang F. Numerical simulation of natural gas non-catalytic partial oxidation reformer, China, Shanghai. International Journal Hydrogen Energy, 2014, vol. 39, pp. 9149-9157.
26. Mevawala C., Jiang Y., Bhattacharyya D. Techno-economic optimization of shale gas to dimethyl ether production processes via direct and indirect synthesis routes, USA, Richland. Applied Energy, 2019, vol. 238, pp. 119-134.

27. Bellotti D., Dierks M., Moellenbruck F., Magistri L., Görner K., Oeljeklaus G. Thermodynamic and economic analysis of a plant for the $\mathrm{CO} 2$ hydrogenation for methanol production, Germany, Essen. E3S Web of Conferences, 2019, vol. 1, pp. 1-7.

28. Zagashvili Yu.V., Levikhin A.A., Kuzmin A.M. Design basics for a threecomponent syngas gas generator. Neftegazokhimiya, 2017, no. 4, pp. 9-16. In Rus.

29. Zagashvili Yu.V., Efremov V.N., Kuzmin A.M. Sposob polucheniya vodorodsoderzhashchego gaza dlya proizvodstva metanola i ustroystvo dlya ego osushchestvleniya [Method of producing a hydrogen-containing gas for producing methanol and device for its implementation]. Patent RF, no. 2632846, 2017.

30. Zagashvili Yu.V., Efremov V.N., Kuzmin A.M. Malotonnazhnaya ustanovka polucheniya metanola [Low-tonnage methanol production unit]. Patent RF, no. 176510, 2018.

31. Zagashvili Yu.V., Kuzmin A.M. Management of the synthesis gas composition for low-tonnage production of methanol. Tekhnologii nefti i gaza, 2018, no. 3, pp. 54-59. In Rus.

32. Bilera I.V., Borisov A.A., Borunov A.B., Kolbanovskij Yu.A., Korolev Yu.M., Rossihin I.V., Troshin I.V. The formation of finely dispersed carbon black in the preparation of synthesis gas under conditions of combustion of methane. Gazokhimiya, 2010, no. 3 (13), pp. 72-78. In Rus.

33. Lugvishchuk D.S., Kulchakovskiy P.I., Mitberg E.I., Mordkovich V.Z. Sazheobrazovanie v protsesse partsialnogo okisleniya metana $\mathrm{v}$ usloviyakh chastichnogo nasyshcheniya parami vody [Soot formation during partial methane oxidation under conditions of partial water vapor saturation]. Neftekhimiya, 2018, vol. 58, no. 3, pp. 324-331.

34. Arutyunov V.S., Strekova L.N. Kinetic features and technological perspectives of the partial oxidation of light alkanes, Russia, Moscow. Russian Journal of Physical Chemistry, 2012, vol. 6, pp. 486-497.

35. Tran L.S., Glaude P.A., Battin-Leclerc F. Experimental study of the structure of laminar premixed flames of ethanol/methane/oxygen/argon. Combustion, Explosion, and Shock Waves, 2013, vol. 49, pp. 11-18.

36. Otchet o NIR. Teoreticheskoe issledovanie rezhimnykh parametrov $i$ oblika novogo generatora sintez-gaza, razrabotka avtomatizirovannoy sistemy kontrolya $i$ upravleniya $i$ konstruktorskoy dokumentatsii generatora [Theoretical investigation of operational parameters and appearance of a new generator of synthesis gas, development of automated system control and generator design documentation]. Available at: http://www.rosrid.ru/ nioktr/JDBZNL5ANBV1BU08S31MDOVM (accessed 28 March 2020).

Received: 28 March 2020.

\section{Information about the authors}

Yuriy V. Zagashvili, Dr. Sc., professor, general director of LLC «HTR».

Aleksey M. Kuzmin, Cand. Sc., associated professor, Baltic State Technical University; general director LLC «GSG»; council of the Russian Academy of Natural Sciences. 